REVIEW ARTICLE

\title{
Management of Infertility-Recommendations in the COVID-19 Era
}

\author{
Sendhil C Arumugam ${ }^{1}$, Jayasree Manivasakan², Syed Habeebullah ${ }^{3}$
}

\begin{abstract}
Worldwide, the incidence of infertility is $15 \%$. In India, the incidence of infertility varies between states ranging between $3.9 \%$ and $16.8 \%$. India has 22-23 million infertile couples, and the total fertility rate has declined from 3.9 in 1990s to 2.3 in 2019. At present, the estimated number of in vitro fertilization cycles countrywide are around 100,000, and it was expected to reach 250,000 by the end of 2020. But with the COVID-19 pandemic, all these treatments have been brought to a sudden halt. Decision to stop all treatment is because SARS CoV2 is a global health pandemic and infertility treatments are usually electively done. Also we need more studies to show that COVID-19 has no effect on newer pregnancies.
\end{abstract}

Keywords: COVID, Cross-training, Freeze-all, Infertility.

SBV Journal of Basic, Clinical and Applied Health Science (2020): 10.5005/jp-journals-10082-02253

\section{INTRODUCTION}

The World Health Organization (WHO) states that one in every four couples in developing nations is affected by infertility. India has 22-23 million infertile couples, and the total fertility rate has declined from 3.9 in 1990s to 2.3 in 2019. At present, the estimated number of in vitro fertilization cycles countrywide is around 100,000 , and it was expected to reach 250,000 by the end of $2020 .^{1}$ As the world faces a pandemic with enormous casualties and an uncertain future for most people, the physicians in reproductive medicine and their patients are faced with newer challenges.

Patients should be informed that considering the rapid community transmission and the lack of symptoms among infected people, frequent visits to the hospital may endanger them and their families. Healthcare workers in fertility centers should understand that high virulence and the sustained surface viability of the virus may cause severe difficulty in keeping the lab surfaces sterile.

Keeping the healthcare workers and their patients a priority, most fertility societies have advised suspension of elective clinical activities. This is highly applicable to infertility workup and procedures. As we have very little information on the effect of COVID-19 on organogenesis (teratogenic ability) and also on perinatal outcome, ${ }^{2}$ it is better to withhold fertility procedures. But if women are in the middle of an intervention or a treatment cycle or for fertility preservation before oncology treatment, treatment cycles can be completed with the full counseling and understanding of the implications by the patients.

\section{Recommendations}

The following guideline will help decrease the chance of acquiring the infection and help conserve essential resource of healthcare workers. ${ }^{3,4}$

\section{Social Distancing}

- All persons entering clinical spaces, both patients and healthcare workers, should be carefully assessed for body temperature and health status.

- Minimize the number of healthcare providers in the clinic.

\begin{abstract}
${ }^{1,3}$ Department of Obstetrics and Gynaecology, Mahatma Gandhi Medical College and Research Institute, Sri Balaji Vidyapeeth, Puducherry, India

${ }^{2}$ Department of Obstetrics and Gynaecology, Sri Manakula Vinayagar Medical College and Hospital, Puducherry, India

Corresponding Author: Sendhil C Arumugam, Department of Obstetrics and Gynaecology, Mahatma Gandhi Medical College and Research Institute, Sri Balaji Vidyapeeth, Puducherry, India, Phone: +91 9485475508, e-mail: senthilcoumarya@mgmcri.ac.in

How to cite this article: Arumugam SC, Manivasakan J, Habeebullah S. Management of Infertility-Recommendations in the COVID-19 Era. J Basic Clin Appl Health Sci 2020;3(2):53-55.

Source of support: Nil

Conflict of interest: None
\end{abstract}

- Minimize number of patients in the reception and patient waiting area when they come in for urgent procedures.

- Follow social distancing by shifting the OPD practice to the telehealth model either using telephone or computer-based face-to-face office-based consultations.

\section{Travel}

- Advise patients to avoid travel to fertility clinics for the sole purpose on non-emergency procedures like egg donation, gestational surrogacy, etc.

- Patients who have had babies delivered through gestational carriers from travel-restricted areas should make alternative care plans for the infants until the travel restrictions are withdrawn.

- For geographically distant patients teleconsultations are recommended to ascertain the need for an appointment.

\section{Clinical Management}

- Plan suspension of all new treatment cycles that include ovulation induction, intrauterine inseminations, in vitro fertilization, and gamete cryopreservation.

(c) The Author(s). 2020 Open Access This article is distributed under the terms of the Creative Commons Attribution 4.0 International License (https://creativecommons. org/licenses/by-nc/4.0/), which permits unrestricted use, distribution, and non-commercial reproduction in any medium, provided you give appropriate credit to the original author(s) and the source, provide a link to the Creative Commons license, and indicate if changes were made. The Creative Commons Public Domain Dedication waiver (http://creativecommons.org/publicdomain/zero/1.0/) applies to the data made available in this article, unless otherwise stated. 
- As there is paucity of data regarding the impact of COVID-19 on pregnancy and fetus, complete cessation of programs that include embryo transfer whether fresh or frozen.

- Urgent care refers to care that is time-sensitive like fertility preservation in impending cancer treatment, medical or surgical.

- Continuation of care to be given to all women who have already been started on ovulation induction regimens or for IVF. This treatment should continue just for a single cycle and cannot be prolonged.

- If oocyte retrieval has been planned, go for the "freeze all" method and cryopreserve all gametes or embryos.

- All nonemergency procedures like tube testing and diagnostic hysteroscopy to be withheld.

\section{Management of Fertility Laboratories}

- Ensure safety of gametes and embryos by employing minimum number of personnel in labs.

- Cross-training of lab personnel and making them come in rotation will help in the event of a staff member being infected, quarantined, or sick.

- In the event of the whole team being quarantined, arrangements should be made to continue keeping the lab safe and transfer of patients to other centers.

- European Society of Human Reproduction and Embryology (ESHRE) guidelines on safe lab practices should be followed and interpersonal interaction in labs should be minimized.

- In the event of incubators or other laboratory spaces used previously for individuals infected with COVID, they should be thoroughly decontaminated before putting it back into service.

- Tissue storage tanks maintenance should continue without interruption.

\section{Psychological Health of Patients and StAFF}

- Fertility care teams should be proactive in providing emotional and psychological support not only to patients but also to all staff involved.

- Patient concerns may be cancellation of cycles/running out of time/impact of COVID on upcoming pregnancies and risk of exposure to COVID during clinic visits.

- Medical staff may be anxious about exposure to COVID, childcare concerns, increased workload, and financial uncertainty.

- Teleconsultation with mental health professionals should be arranged for patients and staff.

\section{Guidance on Recommencing Fertility Treatments ${ }^{5}$}

Once the pandemic stabilizes, we need to arrange the provision for restarting assisted reproductive treatments. ${ }^{5}$ These treatments have to be in line with local regulations. Teleconsultation and triage have an important role in restarting the fertility clinics. Patients, staff, and any other person attending the center need to be triage negative. Each center should adapt the triage questions. These triage questions should be used in the teleconsultation to screen and give appointments to patients. On arrival to the center, they again need to be screened before entering the clinic premises or spaces. If they screen positive to the triage questions either at teleconsultation or on entry, viral screening is recommended.
- High-risk patients, e.g., women with diabetes, patients on immunosuppressants, and patients with lung and liver diseases, should not start ART treatment until it is deemed safe by healthcare authorities.

- All women should be offered a choice to postpone ART treatment and their choices should be documented.

- Patients should be clearly and comprehensively be informed of the risk related to developing COVID in pregnancy.

- All women who have had prior COVID infection should test negative before treatment.

- Manage appointments in such a way that the patients will complete all needed work in a single visit.

- Staff members suspected of infection should be tested in line with the national recommendations.

- All staff turning symptomatic or positive should receive proper advice and go into self-quarantine. They should reattend work once they turn negative with a documented negative RT-PCR or equivalent.

- Contact tracing should be done if a staff member is diagnosed positive.

- The team should be divided into mini teams, undertake crosstraining, and should work in a rotating schedule.

- Restrict patients, staff, and others entering into the clinic premises.

- Routine sanitation of all areas should be continued. COVIDspecific sanitation procedures should be followed if a patient or staff turns positive.

- Special care must be taken to disinfect vaginal probes used during ovarian stimulation and oocyte retrieval.

- Follow good laboratory principles and take extra care not to get exposed to extrafollicular fluid and semen. There should be careful disposal of tissue and stored fluids in closed containers.

- If the patient is suspected/or has infection, after oocyte retrieval a "freeze-all" technique should be followed.

- Patients and staff members should agree and accept to follow a code of conduct, which prohibits them from attending social gatherings and they must affirm it at regular intervals.

These guidelines are as on date. These may change as the understanding of COVID-19 is still evolving. Since our country is in lockdown when this article is being written, routine clinical care may start only after ICMR recommendations. So, we need to update our knowledge on a regular basis with national and international fertility societies to keep up with the changing guidelines.

\section{References}

1. Sabahat $\mathrm{R}$, Omar $\mathrm{SA}$. The huge burden of infertility in India - Are we crumbling underneath? Glob J Reprod Med 2018;5(3):555670. DOI: 10.19080/GJORM.2018.05.555670.

2. Schwartz DA. An analysis of 38 pregnant women with COVID-19, their newborn infants, and maternal-fetal transmission of SARS-CoV-2: maternal coronavirus infections and pregnancy outcomes. Arch Pathol Lab Med 2020. DOI: 10.5858/arpa.2020-0901-SA.

3. ASRM. Patient Management and Clinical Recommendations during the Coronavirus(COVID-19) Pandemic (as of March 17, 2020).

4. ESHRE. Assisted reproduction and COVID-19. A statement from ESHRE for phase 1 - Guidance on fertility services during pandemic. Published on 2 April 2020 (last revision 17 April).

5. ESHRE. ESHRE guidance on recommencing ART treatments. Document prepared by the ESHRE COVID-19 Working Group Published on the ESHRE website. Date of publication: 23/04/2020 Last update: 05/05/2020 (addition clarification on triage and testing). 


\section{APPENDIX: Triage Questions}

- Have you been sick in the last 2 weeks?

- Do you have fever $\left(>37.5^{\circ} \mathrm{C}\right)$ ?

- Do you have you cough at present?

- Do you have a sore throat?

- Have you lost your sense of smell or taste?

- Have you been in contact with somebody who had cough/fever or sore throat?

- Have you recently traveled to a red zone or a containment zone?

- Do you work in a hospital/nursing home or healthcare facility?

- Have you been in contact with somebody who has COVID-19?

- Have you been diagnosed with COVID-19?

- Do you live in a household with somebody who has been diagnosed with COVID-19?

- If you have been COVID-19 positive and recovered, do you have certified medical evidence of clearance?

- Do you have a severe comorbid medical condition like diabetes, respiratory disease, chronic kidney disease, etc.? 